OPEN ACCESS

Edited by:

Laszlo Csiba,

University of Debrecen, Hungary

Reviewed by:

Ronen R. Leker,

Hadassah University Hospital, Israel

Ru-Lan Hsieh,

Shin Kong WHS Memorial Hospital,

Taiwan

*Correspondence:

Thanh G. Phan

thanh.phan@monash.edu

Specialty section:

This article was submitted to Stroke,

a section of the journal

Frontiers in Neurology

Received: 17 November 2016 Accepted: 13 February 2017

Published: 28 February 2017

Citation:

Phan TG, Chen J, Beare R, Ma H, Clissold B, Van Ly J, Srikanth V and the VISTA-ICH Collaboration (2017) Classification of Different Degrees of

Disability Following Intracerebral Hemorrhage: A Decision Tree Analysis from VISTA-ICH Collaboration.

Front. Neurol. 8:64.

doi: 10.3389/fneur.2017.00064

\section{Classification of Different Degrees of Disability Following Intracerebral Hemorrhage: A Decision Tree Analysis from VISTA-ICH Collaboration}

\author{
Thanh G. Phan ${ }^{1 *}$, Jian Chen ${ }^{2}$, Richard Beare ${ }^{2}$, Henry Ma $^{1,2}$, Benjamin Clissold ${ }^{1,2}$, \\ John Van Ly ${ }^{1,2}$, Velandai Srikanth ${ }^{1,3}$ and the VISTA-ICH Collaboration
}

\begin{abstract}
${ }^{1}$ Neurosciences, Monash Health, Melbourne, VIC, Australia, ${ }^{2}$ Department of Medicine, School of Clinical Sciences, Monash University, Clayton, VIC, Australia, ${ }^{3}$ Department of Medicine, Central Clinical School, Monash University, Frankston, VIC, Australia
\end{abstract}

Background and purpose: Prognostication following intracerebral hemorrhage (ICH) has focused on poor outcome at the expense of lumping together mild and moderate disability. We aimed to develop a novel approach at classifying a range of disability following $\mathrm{ICH}$.

Methods: The Virtual International Stroke Trial Archive collaboration database was searched for patients with $\mathrm{ICH}$ and known volume of $\mathrm{ICH}$ on baseline CT scans. Disability was partitioned into mild [modified Rankin Scale (mRS) at 90 days of 0-2], moderate $(m R S=3-4)$, and severe disabilities (mRS $=5-6)$. We used binary and trichotomy decision tree methodology. The data were randomly divided into training (2/3 of data) and validation ( $1 / 3$ data) datasets. The area under the receiver operating characteristic curve (AUC) was used to calculate the accuracy of the decision tree model.

Results: We identified 957 patients, age $65.9 \pm 12.3$ years, $63.7 \%$ males, and $\mathrm{ICH}$ volume $22.6 \pm 22.1 \mathrm{ml}$. The binary tree showed that lower $\mathrm{ICH}$ volume $(<13.7 \mathrm{ml})$, age ( $<66.5$ years), serum glucose $(<8.95 \mathrm{mmol} / \mathrm{l})$, and systolic blood pressure $(<170 \mathrm{~mm} \mathrm{Hg})$ discriminate between mild versus moderate-to-severe disabilities with AUC of 0.79 (95\% $\mathrm{Cl}$ 0.73-0.85). Large ICH volume (>27.9 ml), older age (>69.5 years), and low Glasgow Coma Scale $(<15)$ classify severe disability with AUC of 0.80 (95\% Cl 0.75-0.86). The trichotomy tree showed that $\mathrm{ICH}$ volume, age, and serum glucose can separate mild, moderate, and severe disability groups with AUC 0.79 (95\% Cl 0.71-0.87).

Conclusion: Both the binary and trichotomy methods provide equivalent discrimination of disability outcome after $\mathrm{ICH}$. The trichotomy method can classify three categories at once, whereas this action was not possible with the binary method. The trichotomy method may be of use to clinicians and trialists for classifying a range of disability in $\mathrm{ICH}$.

Keywords: decision trees, intracerebral hemorrhage, outcome, disability evaluation, stroke 


\section{INTRODUCTION}

The incidence of intracerebral hemorrhage (ICH) is estimated at 24.6 per 100,000 per year (1). Despite the advances in stroke prevention and management, a recent meta-analysis has suggested that the incidence of ICH and its associated median 1 month mortality of $40 \%$ have not changed between 1980 and 2008 (1) (mortality is much lower in Japan). There have been many models (2-6) for prediction of poor outcome following ICH. These models emphasized the importance of the volume of the hematoma and the Glasgow Coma Scale (GCS) (5). Importantly, these models have focused mostly on predicting mortality or poor outcome (severe disability) (2, 4, 7-10). An earlier review in 2005 of prediction models for mortality showed that they have high specificity but low sensitivity and consequently do not perform well as a clinical triaging tool (2). A more recent systematic review a decade later revealed that tools based on ICH score had excellent ability to discriminate mortality with area under ROC curve between 0.8 and 0.87 (11). However, it is less certain if these tools can discriminate the outcome over the range of disabilities.

For prognostication, clinicians want to classify mild and severe disability outcomes. Because of the way the outcome data are dichotomized from the Rankin scale of disability, good outcome is buried in a much larger group comprising good and intermediate outcome $(2,3,7)$. However, clinicians and trialists may also want to know about patients with mild, moderate, or severe disability (12). Surprisingly, few studies have focused on classification of mild disability (10). One may speculate that inclusion of patients who are likely to have mild disability in clinical trials may have been the reasons for the lack of positive results in these randomized control trials $(13,14)$.

Most models have been developed using regression-based methods such as logistic regression and only two studies (with small sample size) have used classification and regression tree (CART) analysis $(15,16)$. Regression-based methods are used for hypothesis testing and generation of predictive models. These models assume that all of the variables are required at once to formulate an accurate prediction.

Such approaches assume that all of these beta coefficients are required at once to formulate a model. Due to the sequential nature of typical clinical reasoning, some predictors will be used and some will be left out (see Figures 1 and 2). This would make some of the elements of any model from regression analysis superfluous. By contrast, a decision tree method generates a logical flow diagram that resembles a tree (17). This triangulated diagram, with repeated partitioning of the original data into smaller groups (nodes) on a yes or no basis, resembles clinical reasoning.

In order to develop a decision tree model, we sought a much larger dataset than previous studies, through the Virtual International Stroke Trial Archive (VISTA) (18). This archive contains data from available trials on ICH. Our aim was to use binary and trichotomy decision trees to predict mild, moderate, and severe disability outcomes at 3 months following ICH.

\section{MATERIALS AND METHODS}

Virtual International Stroke Trial Archive contains data from clinical trials including both ischemic stroke and ICH trials (18). Patients or their legal surrogates had signed informed consents for participation in clinical trials. The data are released in deidentified manner so that the trials and treatment allocations are not known. As such the name of the trials and the dates in which the patients were recruited are not provided here. We searched VISTA records for the patients with ICH. The following fields were used for extraction of imaging data: volume of ICH, intraventricular hemorrhage, midline (septum pellucidum) shift, location (basal ganglia, lobar and infratentorial hemorrhage); baseline clinical data: GCS, physiological variables (systolic blood pressure, blood sugar level), demographic data (age, gender), risk factors (hypertension, diabetes, antiplatelet drugs), and 3 months outcome data [modified Rankin scale (mRS)].

\section{Decision Tree Analyses}

We used recursive partitioning (known as rpart, which is a free version of CART and available from $\mathrm{R}$ foundation, http://cran.rproject.org/web/packages/rpart/rpart.pdf) to perform binary decision tree analyses. The binary term here refers to splitting the data into two major outcomes of interest: such as mild versus moderate to severe disabilities. The method uses a splitting rule built around the notion of "purity." A node in the tree is defined as pure when all the elements belong to one class. When there is impurity in the node, a split occurs to maximize reduction in "impurity." In some cases, the split may be biased toward attributes that contain many different ordinal levels or scales (19). Thus, the selection of an attribute as the root node may vary according to the splitting rule and the scaling of the attribute (19).

By contrast, the term trichotomy decision tree analyses refer to trees where the data are partitioned into three major outcomes of interest: mild, moderate, and severe disabilities. In this paper, the term trichotomy is preferred in order to avoid confusion with ordinal regression analysis. This analysis is performed using rpartScore (20). In this analysis, attributes with greater than $10 \%$ missing data are not used for the predictive model (20).

\section{Binary Decision Tree Models}

In model 1, the variables included GCS in addition to age, gender, ICH volume at baseline, glucose, and systolic blood pressure. This step was taken in an attempt to replicate part of the ICH score (4) (with the exception of location of ICH). In model 2, we used baseline NIHSS instead of GCS and keeping the other predictors to be the same. The NIHSS was chosen here because it has recently been assessed for use in ICH (21).

\section{Trichotomy Decision Tree Models}

The mRS ranges from 0 (no disability) to 6 (death). The mRS of 1 equates to minimal disability, 2 to mild disability, 3 to moderate disability, 4 to moderately severe disability, and 5 to severe disability and bed ridden. We partition the mRS as followed good (mRS 0-2), moderate (mRS 3-4), and severe disability and death (mRS 5-6) to keep the ordinal structure of the scale (22). In this analysis, we did not consider it clinically useful to separate 


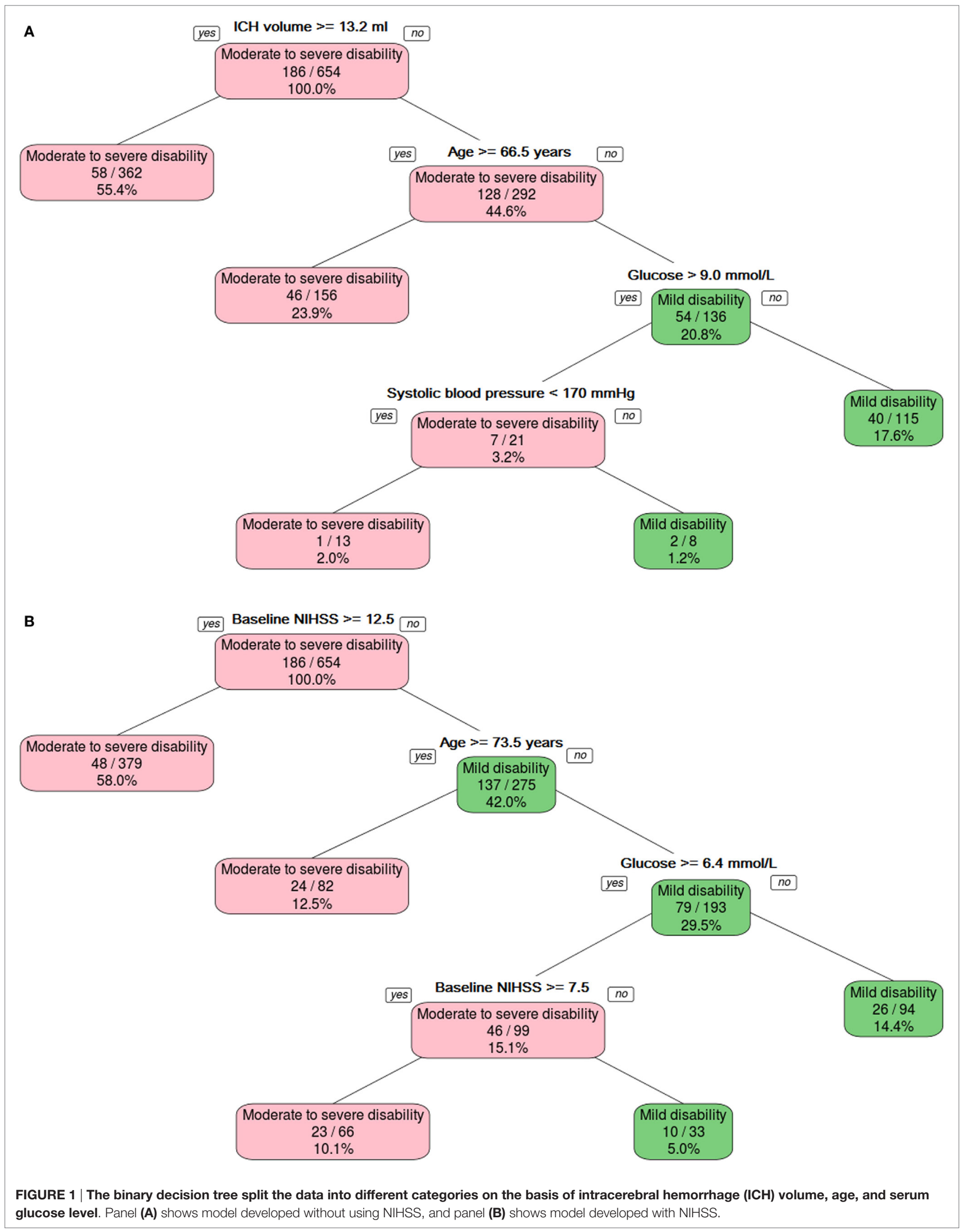




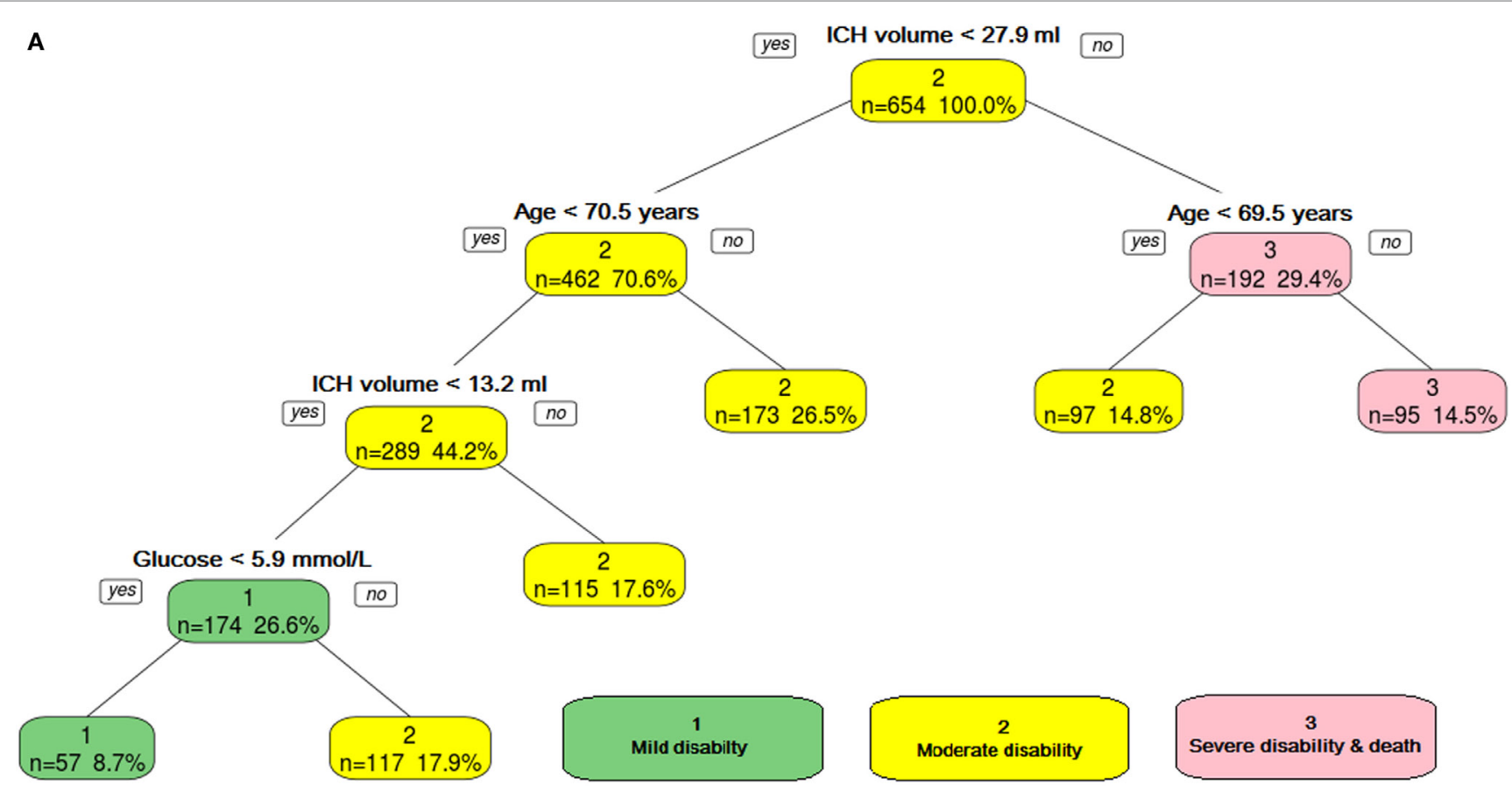

B

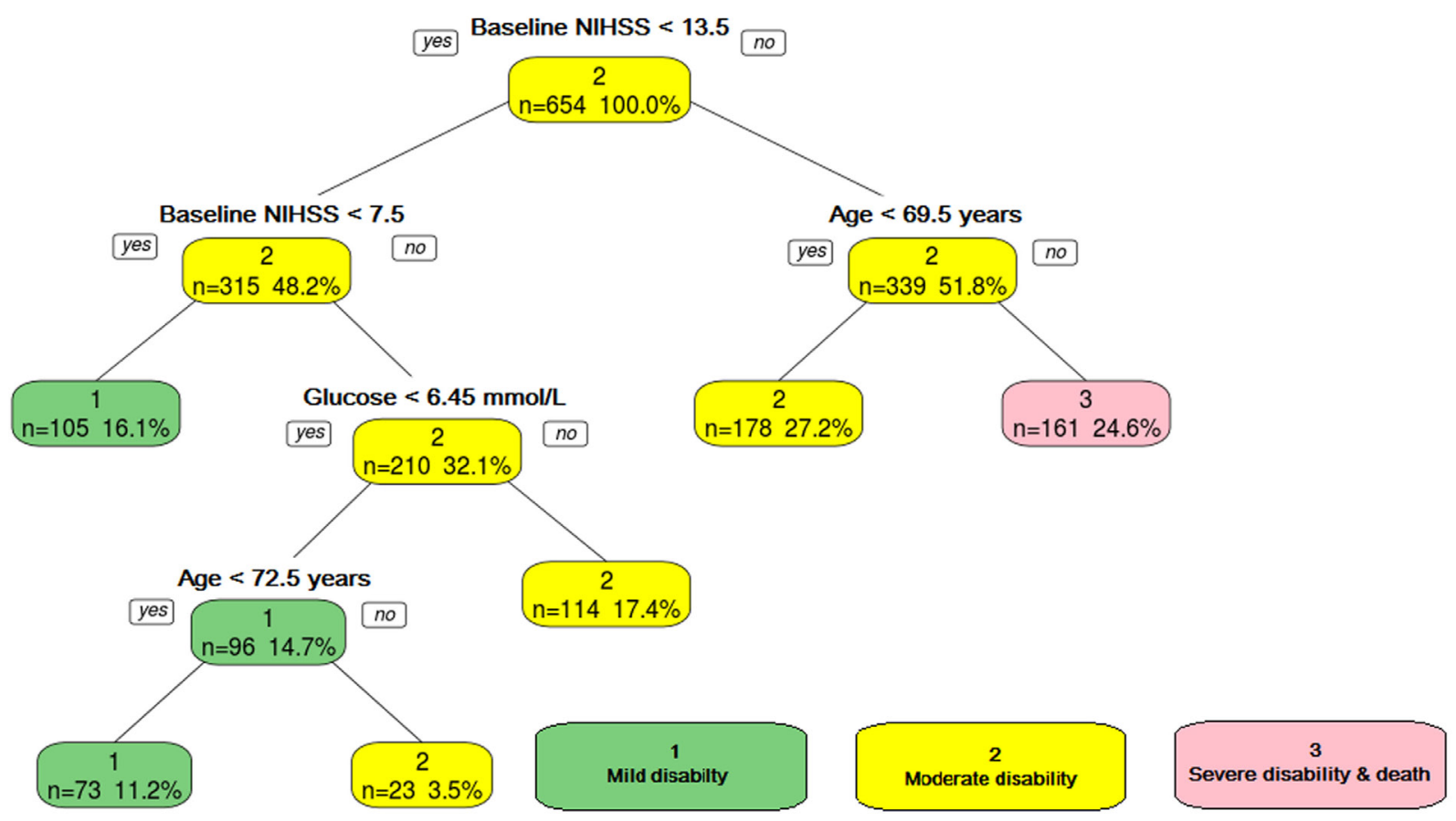

FIGURE 2 | The ordinal decision tree split the data into different categories on the basis of intracerebral hemorrhage (ICH) volume, age, and serum glucose level. Panel (A) shows model developed without using NIHSS, and panel (B) shows model developed with NIHSS. Category 1-mild disability, Category 2-moderate, and Category 3-poor disability.

outcome using the full range of the mRS since it would create many partitions with poor reproducibility. It has been suggested that $\mathrm{mRS}=4$ should be part of severe disability. We have also explored this analysis with a different partition of the mRS: good (mRS $0-2)$, moderate ( $\mathrm{mRS} 3$ ), and severe disability and death (mRS 4-6). 
In model 3, we combined models 1 and 2 . As such, both variables such as baseline NIHSS and GCS were used together in conjunction with the other demographic and physiologic variables.

\section{Validation}

For the purpose of validation, we randomly extracted two-thirds of the original data to train the models and used the remaining one-third for validation. The discriminating ability of the models was assessed using the area under the receiver operating characteristic curve (AUC) and interpreted using the guidelines set by Hosmer and Lemeshow (23). An AUC of 0.5 is classified as no better than by chance; $0.6-0.69$ provides poor discrimination; 0.7-0.79 provides acceptable (fair) discrimination; 0.8-0.89 provides good (excellent) discrimination; and $0.9-1.0$ provides outstanding discrimination (23). The trichotomy decision tree models were assessed using the area under the ordinal ROC curve function. The differences in the AUC between the training and validation ROC curves were compared using the $Z$-score.

\section{RESULTS}

\section{Patient Demographics}

Using the search criteria described in Section "Materials and Methods," we identified 1,371 patients. From these data, there were 957 patients with complete data on important covariates such as age, gender, ICH volume, baseline NIHSS, baseline GCS, and systolic blood pressure. The mean age was $65.9 \pm 12.3$ years (63.7\% males). The baseline ICH volume was $22.6 \pm 22.1 \mathrm{ml}$, and the median was 15.2 (IQR 7.7-30.5).

The mean GCS was $13.7 \pm 1.8$ and the median was 15 (IQR 13-15). The mean baseline NIHSS was $13.7 \pm 5.8$ and the median NIHSS was 14 (IQR 9-18).

The frequency of hypertension was $64.9 \%$ (data available on 842 of 957 patients) and diabetes was 13.9\% (data available on 842 of 957 patients). The serum glucose was $8.7 \pm 13.4 \mathrm{mmol} / \mathrm{l}$ (data available in 890 of 957 patients) and systolic blood pressure was $176.6 \pm 30.1 \mathrm{~mm} \mathrm{Hg}$. Patients had CT scan early after onset with the average time to CT scan being $3.7 \pm 1.2 \mathrm{~h}$. Too few patients had data recorded on location of ICH (31\%) or presence of intraventricular hemorrhage/IVH (37.7\%) and midline shift $(61 \%)$ to include these variables in our analyses.

\section{Relationship between NIHSS, GCS, and ICH Volume}

There was a positive correlation between NIHSS at baseline and the ICH volume (Spearman rho 0.62, 95\% bootstrap CI $0.57-0.67)$. There was negative correlation between NIHSS and GCS (spearman rho $-0.60,95 \%$ bootstrap CI -0.65 to -0.54 ), and between GCS and ICH volume (spearman rho $-0.45,95 \%$ bootstrap CI -0.510 to -0.39$)$.

\section{Prediction of Mild Disability with Binary Decision Tree-Model 1 (Focusing on GCS, ICH Volume, and Demographic and Physiologic Variables)}

The binary decision tree (Figure 1A) showed that lower ICH volume $(<13.2 \mathrm{ml})$ followed by younger age ( $<66.5$ years), serum glucose $(\leq 9.0 \mathrm{mmol} / \mathrm{l})$, and systolic blood pressure $(<170 \mathrm{~mm}$ $\mathrm{Hg}$ ) discriminated between mild disability (mRS $0-2$ ) versus moderate-to-severe disability or death (mRS 3-6). The AUC for predicting mild disability was 0.79 (95\% CI $0.73-0.85$ ) in the training data and 0.66 (95\% CI $0.57-0.75)$ in the validation data. The $Z$-score for comparison between the training and validation binary decision trees was statistically different, $2.27(p=0.02)$.

The binary decision tree (Figure 1B) showed higher baseline NIHSS $(>12)$, older age $(\geq 73.5)$, and blood sugar level ( $>6.4 \mathrm{mmol} / \mathrm{l}$ ) discriminated between mild disability (mRS 0-2) versus moderate-to-severe disability or death (mRS 3-6). The AUC for predicting mild disability was in the training in validation data were 0.82 (95\% CI $0.76-0.87)$ and 0.77 (95\% CI $0.69-0.85)$. The $Z$-score for comparison between the training and validation binary decision trees was not statistically different, 0.94 $(p=0.3)$.

\section{Model 1 (Focusing on GCS, ICH Volume, and Demographic and Physiologic Variables)-Prediction of Disability with Trichotomy Decision Tree}

The trichotomy decision tree (Figure 2A) showed that $\mathrm{ICH}$ volume, age, and serum glucose helped to separate the three classes of outcome. The AUC was 0.79 (95\% CI 0.71-0.87) for the training data and 0.68 (95\% CI $0.53-0.83)$ for the validation data.

The trichotomy decision tree (Figure 2B) showed that high baseline NIHSS $(\geq 14)$ and age $(\geq 69.5)$ defined the group with severe disability; if the age is below 69.5 years old, then it defines the group with moderate disability. The group with mild disability is defined as having lower baseline NIHSS $(<8)$. Those patients with higher baseline NIHSS $(\geq 8)$ and higher blood sugar level ( $\geq 6.5 \mathrm{mmol} / \mathrm{l}$ ) have moderate disability. The AUC was $0.73(95 \%$ CI $0.67-0.79$ ) for the training data and 0.68 (95\% CI 0.58-0.79) for the validation data.

\section{Prediction with Binary Decision Tree- Model 2 (Focusing on Baseline NIHSS, ICH Volume, and Demographic and Physiologic Variables)}

The binary decision tree showed that NIHSS $(<12.5)$, followed by age $(<73.5$ years old) and serum glucose $(<6.35 \mathrm{mmol} / \mathrm{l})$ discriminated between mild and moderate-to-severe disability. The AUC for predicting good outcome in the training group was 0.82 (95\% CI 0.76-0.87). The AUC in the validation group was 0.77 (95\% CI 0.69-0.85). The Z-score was 0.94 ( $p=0.3$ ), indicating no statistically significant difference between the training and validation binary decision trees.

\section{Prediction with Trichotomy Decision Tree-Model 2 (Focusing on Baseline NIHSS, ICH Volume, and Demographic and Physiologic Variables)}

The trichotomy decision tree (Figure 2) showed NIHSS, age, and serum glucose helped to separate the three classes of outcome. The AUC for the training data was 0.73 (95\% CI 0.067-0.79). The 
AUC in the validation group was 0.68 (95\% CI $0.58-0.79)$. The $Z$-score was $0.76(p=0.4)$, indicating no statistically significant difference between the training and validation trichotomy decision trees.

\section{Model 3 (Baseline NIHSS, GCS, ICH Volume, and Demographic and Physiologic Variables)}

This model performed the same as model 2 above.

We have also analyzed the data with a different partition of disability: mild (mRS 0-2), moderate ( $\mathrm{mRS} 3$ ), and severe (mRS 5-6). For the model with covariates such as NIHSS, ICH volume, and demographic and physiologic variables, the AUC for the training data was 0.61 (95\% CI 0.53-0.69). For the model with covariates such as NIHSS, GCS, ICH volume, and demographic and physiologic variables, the AUC for the training data was 0.54 (95\% CI 0.42-0.66).

\section{Prediction of Severe Disability and Death-Model 1 (Focusing on GCS, ICH Volume, and Demographic and Physiologic Variables)}

The binary decision tree showed that higher ICH volume $(>27.9 \mathrm{ml})$ followed by older age $(>69.5$ years old) and low GCS $(<15)$ discriminated between severe (mRS 5-6) and mildto-moderate disability (mRS $0-4)$. The AUC was 0.80 (95\% CI $0.75-0.86)$ in the training data and 0.79 (95\% CI $0.70-0.88)$ in the validation data (Figure 3A). The $Z$-score for comparison was $0.17(p=0.9)$, indicating no difference.

\section{Combining NIHSS and GCS}

The binary decision tree showed that higher NIHSS $(\geq 13.5)$, followed by older age ( $\geq 77.5$ years old) and larger ICH volume $(44.6 \mathrm{ml})$ discriminated between severe disability and death versus mild-to-moderate disability (Figure 3B). The AUC was 0.84 (95\% CI $0.79-0.89)$ in the training data and 0.78 (95\% CI $0.69-0.87)$ in the validation data. The $Z$-score for comparison was $1.16(p=0.2)$, indicating no difference.

\section{DISCUSSION}

In this exploratory study, we have evaluated the use of trichotomy decision tree method for classifying outcome in $\mathrm{ICH}$. These models were developed from clinical and imaging information that would be available at the time of patient presentation to the hospital. The trichotomy and binary methods are simple in both concept and usage, requiring very few attributes. These findings offer several methodological approaches to defining the group with moderate disability and who may benefit from participating in clinical trial.

\section{ICH Volume, GCS, and NIHSS}

In this study, we had performed decision tree modeling with ICH volume and GCS rather than NIHSS; GCS had been used for developing prognostic model of mortality in patients with ICH
$(4,5)$. Compared to NIHSS, GCS did not remain in the model for classifying mild disability probably because these patients would have GCS 14 or greater (see Figure 1) (15). By contrast, NIHSS provided a better partition for the data than both $\mathrm{ICH}$ volume and GCS for the prediction of mild disability (models 2 and 3). There were no differences in discrimination between models based on NIHSS and ICH volume and GCS in the models for severe disability.

\section{Comparison with Other Models}

Our models have not relied on empirical threshold such as predefined ICH volume but are data driven $(4,5,16)$. Previous models have empirically used ICH volume of $30 \mathrm{ml}$ or greater to differentiate between severe disability and death (mRS 0-4) $(2,4,5)$. In our study, an ICH volume approximately less than $30 \mathrm{ml}$ was compatible with either mild or moderate disability, whereas an ICH volume less than $13 \mathrm{ml}$ and glucose level less than $5.9 \mathrm{mmol} / \mathrm{l}$ occurred predominantly in patients with mild disability.

In this study, we were limited to data that have been entered into VISTA, and therefore, we do not have complete data on all variables that would be present at the time of patient's presentation to hospital. Furthermore, we are limited in that the analysis in our project should not overlap with other VISTA projects. The aim here is to provide an exploration of the trichotomy decision tree method, and hence, the results of this analysis should be seen as an exploration and not a definitive predictive tool. The paucity of coded data on location of ICH and IVH extension meant that we cannot extend our analysis into the modifying effect of these variables on outcome in a way that has been done with ICH score (4). In the recent factor VIIa trial, there were $1.3 \%$ of patients with infratentorial ICH in the treatment arm and $2.6 \%$ in the placebo arm (13). It is possible that even if these data were available, there would not be enough of these cases to make inference about impact of ICH location. This low frequency may have been due to the requirement in such trial to exclude patients with plan for surgical evacuation within $24 \mathrm{~h}$ (13).

Similarly, data on other potential predictor of ICH growth, such as the spot sign, were not available for analysis (24). Our findings should be seen as example of what can be achieved with advancement in decision tree methods rather than finalized model for prediction. Consequently, we would seek to collaborate with other groups regarding application of such methods in the analysis of ICH outcome.

\section{Implications of ICH Volume in Clinical Trial Design}

Our findings may have implication for clinical trial design, given they suggest that patients with ICH volume less than $13 \mathrm{ml}$ are likely to have mild disability at 90 days. It is possible that results from the recent clinical trials may have occurred because they have included many cases of ICH with small volume (and which would have a good outcome regardless of treatment) $(12,13,25)$. The recombinant factor VII trial had an average ICH volume $24 \pm 26$ and $23 \pm 26 \mathrm{ml}$ in the two treatment arms compared to $22 \pm 24 \mathrm{ml}$ in the placebo arm (13). In the recent trial of 


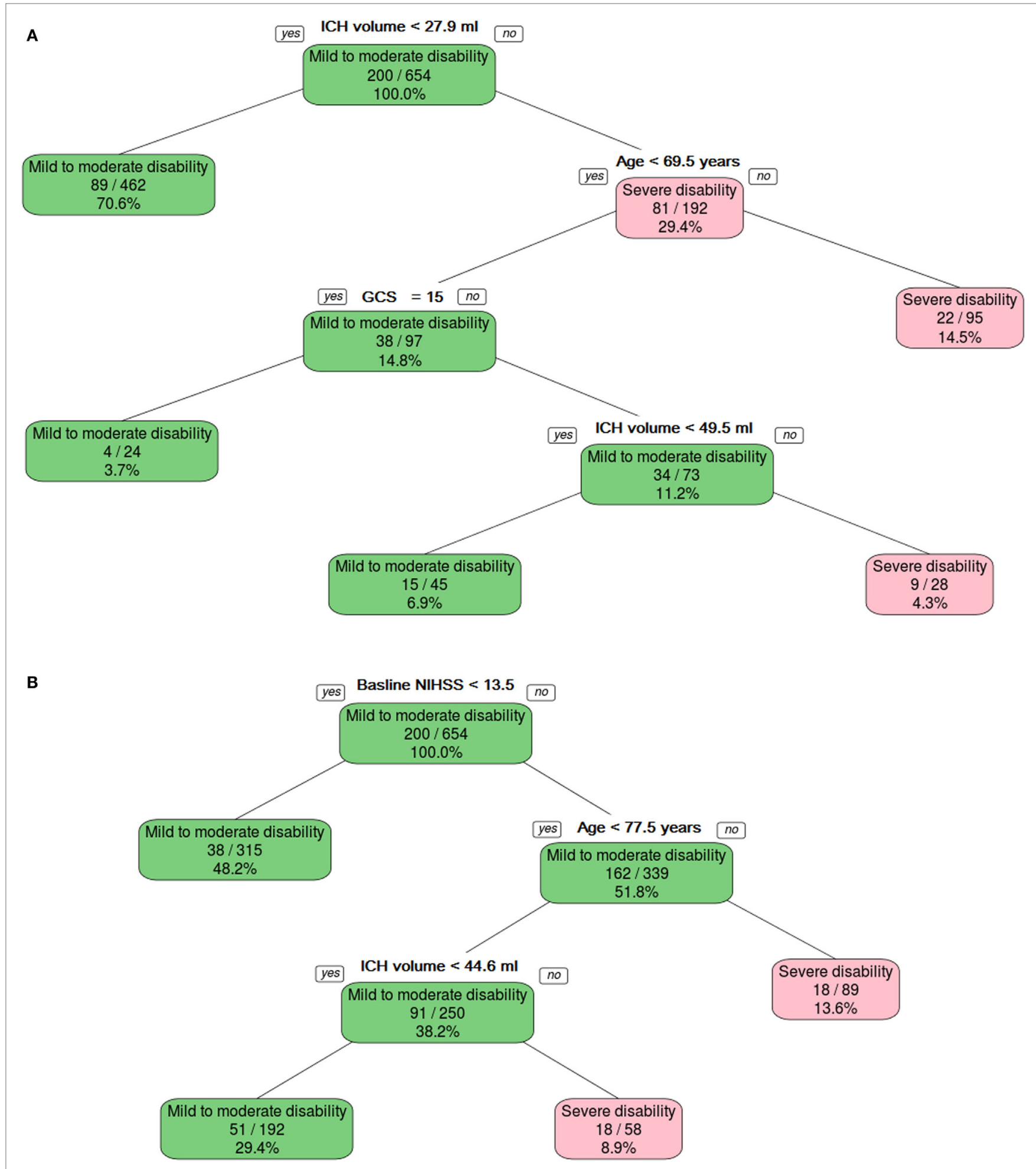

FIGURE 3 | The binary decision tree split the data into different categories on the basis of intracerebral hemorrhage (ICH) volume, age, and Glasgow Coma Scale (GCS). Panel (A) shows model developed without using NIHSS, and panel (B) shows model developed with NIHSS.

intensive blood pressure lowering in acute ICH, 39\% of the treatment arm and $42 \%$ of the placebo arm had ICH volume less than $15 \mathrm{ml}(12)$.

\section{Decision Trees}

Decision trees have been used in machine learning tasks since the 1960s (17) but have been used sparingly in stroke (particularly 
ICH) for model development $(15,16)$. This methodology offers the advantage over standard regression methods in that it tolerates certain degree of missing number because the data are splitted using the available information for that attribute to calculate the Gini index (rather than the entire cohort). Despite this advantage, it is uncertain how much missing data the method would tolerate or whether it biases the results toward those cases that contain these data on these attributes. As such, we have conservatively chosen to avoid including attributes such as ICH location, IVH, and midline shift, where there are greater than $10 \%$ missing data. By contrast, attribute such as serum glucose has $7 \%$ missing data, but this number is with acceptable limit for the analyses. A potential disadvantage of the splitting rule used here is that it may be biased toward attributes that contain many different levels or scales. Thus, the selection of an attribute such as the root node (at the first split) may vary according to the splitting rule and the scaling of the attribute. Coincidentally in this case, the selection of ICH volume such as the root node corresponds to how we would design a clinical pathway and with previous information in the literature on the importance of ICH volume (2).

Decision tree methodology offers a second advantage over regression methods in that it resembles clinical reasoning and without the need to use equations or remembering the weights for each variable. The trade-off is that the user needs to remember the order of each variable in the partition. One may want to develop mnemonics to aid memorizing the order of the attributes. For the attributes in Figure 1A containing ICH volume, age, glucose, and systolic blood pressure, one may develop the mnemonics "I see Angels and Gods in the Sky" as a way to predict moderate and severe disability. In this study, the attributes are easy to remember as they are attributes that have been identified by other investigators to be important in ICH outcome $(2,4,7-10)$. In certain situations, the outcome may be defined at the first, second, or third partitions of the tree. By contrast, models from regression methods attempt to use simultaneously all the attributes to arrive at prediction. Such redundancy in the attributes (as use in regression equation) can be seen in our decision trees where patients with very large ICH volume or high NIHSS are defined to have a poor outcome regardless of other variables.

\section{Trichotomy Decision Tree}

The trichotomy decision tree method has not been used for clinical prediction rule in the stroke literature. The recent incorporation of this method in the R statistical package 2012 may be the reason why this has been the case (20). We were able to delineate the group who have moderate outcome by using the ability of trichotomy decision tree to split the data into three classes or more and without sacrificing accuracy AUC. A more cumbersome approach would be to perform sequential binary decision trees.

The success of the trichotomy decision tree points to a major advantage over regression method such as ordinal regression. That method uses a proportional odds model for analysis, which treats the odds of moving from one category to the other to be the same, i.e., the beta coefficient is constant among categories. That type of regression model applies the "parallel regression assumption" and may not be valid when the assumption of proportional odds does not hold true. In practice, this ideal situation is not always possible in stroke research and researchers have suggested alternative strategy such as the partial proportional odds model (with adjustment made for predictor variables which do not follow this assumption). Some investigators have suggested the combined use of ordinal regression and linear discriminant analysis to derive a prediction model $(26,27)$.

\section{Limitations}

A limitation of this study is that it assumes mortality is due to disease and does not take into account variation in clinical practices such as do not resuscitate orders (28). The frequency of use of such orders impacts on mortality in ICH. Furthermore, the frequency of intensive blood pressure lowering in these trials was not known as the trials in this study were performed prior to the recent intensive blood pressure lowering trial (12). While this may be a limitation of this study, our finding that higher blood pressure is an attribute for prediction of poor outcome is in support of investigators evaluating the role of blood pressure lowering in ICH (12). Our study can be criticized for including subjects with mild stroke given that the median GCS was 13.7. However, the mean NIHSS of 13.7 suggests that these patients have moderately severe stroke deficit (defined as NIHSS 8-15) (29). Finally, there has been discussion on appropriate partition of the Rankin disability scale, particularly with regard to the threshold for moderate disability on the mRS. Based on recommendations to keep the ordinal structure of the mRS, we had chosen the partition as mild (mRS 0-2), moderate (mRS 3-4), and severe disability and death (mRS 5-6) (22). This partition severe disability and death as mRS 5-6 had been used in stroke trials $(30,31)$ and studies on prediction (32) and is consistent with recommendation by panel of experts (33). Nevertheless, we had also performed the analysis with the revised threshold for moderate disability as mRS equals to 3 . Using this revised definition, severe disability and death correspond to mRS 4-6. However, the trichotomy decision tree analysis performed less well using the new threshold, suggesting our original selection for partitioning the $\mathrm{mRS}$ as appropriate.

\section{CONCLUSION}

Decision tree methods can produce models with fair to excellent discrimination for disability outcome. We have shown several methodological approaches to identifying good and poor outcome groups. Both the binary and trichotomy methods provide equivalent discrimination of disability outcome after ICH. The trichotomy method can classify three categories at once, whereas this action was not possible with the binary method. These methods may be of use in clinical trial design and in re-evaluation of other trials for possible signals of efficacy (14).

\section{AUTHOR CONTRIBUTIONS}

Study concept and design: TP. Acquisition of data: TP and VISTAICH collaborators. Analysis and interpretation of data: TP, JC, and RB. Drafting of the manuscript: TP, VS, JC, RB, JL, BC, and HM. Critical revision of manuscript for intellectual content: all the authors. Statistical analysis: TP, JC, and RB. 


\section{ACKNOWLEDGMENTS}

The authors thank the VISTA investigators and the data custodian for their help with this study.

\section{FUNDING}

VS received a NHMRC/Heart Foundation Career Development Fellowship. This funding source had no role in study design,

\section{REFERENCES}

1. van Asch CJ, Luitse MJ, Rinkel GJ, van der Tweel I, Algra A, Klijn CJ. Incidence, case fatality, and functional outcome of intracerebral haemorrhage over time, according to age, sex, and ethnic origin: a systematic review and meta-analysis. Lancet Neurol (2010) 9:167-76. doi:10.1016/ S1474-4422(09)70340-0

2. Ariesen MJ, Algra A, van der Worp HB, Rinkel GJ. Applicability and relevance of models that predict short term outcome after intracerebral haemorrhage. J Neurol Neurosurg Psychiatry (2005) 76:839-44. doi:10.1136/ jnnp.2004.048223

3. Phan TG, Koh M, Vierkant RA, Wijdicks EF. Hydrocephalus is a determinant of early mortality in putaminal hemorrhage. Stroke (2000) 31:2157-62. doi:10.1161/01.STR.31.9.2157

4. Hemphill JC III, Bonovich DC, Besmertis L, Manley GT, Johnston SC. The ICH score: a simple, reliable grading scale for intracerebral hemorrhage. Stroke (2001) 32:891-7. doi:10.1161/01.STR.32.4.891

5. Broderick JP, Brott TG, Duldner JE, Tomsick T, Huster G. Volume of intracerebral hemorrhage. A powerful and easy-to-use predictor of 30-day mortality. Stroke (1993) 24:987-93. doi:10.1161/01.STR.24.7.987

6. Chen HS, Hsieh CF, Chau TT, Yang CD, Chen YW. Risk factors of inhospital mortality of intracerebral hemorrhage and comparison of $\mathrm{ICH}$ scores in a Taiwanese population. Eur Neurol (2011) 66:59-63. doi:10.1159/ 000328787

7. Godoy DA, Pinero G, Di Napoli M. Predicting mortality in spontaneous intracerebral hemorrhage: can modification to original score improve the prediction? Stroke (2006) 37:1038-44. doi:10.1161/01.STR.0000206441. 79646.49

8. Ruiz-Sandoval JL, Chiquete E, Romero-Vargas S, Padilla-Martinez JJ, Gonzalez-Cornejo S. Grading scale for prediction of outcome in primary intracerebral hemorrhages. Stroke (2007) 38:1641-4. doi:10.1161/ STROKEAHA.106.478222

9. Parry-Jones AR, Abid KA, Di Napoli M, Smith CJ, Vail A, Patel HC, et al. Accuracy and clinical usefulness of intracerebral hemorrhage grading scores: a direct comparison in a UK population. Stroke (2013) 44:1840-5. doi:10.1161/ STROKEAHA.113.001009

10. Rost NS, Smith EE, Chang Y, Snider RW, Chanderraj R, Schwab K, et al. Prediction of functional outcome in patients with primary intracerebral hemorrhage: the FUNC score. Stroke (2008) 39:2304-9. doi:10.1161/ STROKEAHA.107.512202

11. Mattishent K, Kwok CS, Ashkir L, Pelpola K, Myint PK, Loke YK. Prognostic tools for early mortality in hemorrhagic stroke: systematic review and meta-analysis. J Clin Neurol (2015) 11:339-48. doi:10.3988/jcn. 2015.11.4.339

12. Anderson CS, Heeley E, Huang Y, Wang J, Stapf C, Delcourt C, et al. Rapid blood-pressure lowering in patients with acute intracerebral hemorrhage. $\mathrm{N}$ Engl J Med (2013) 368:2355-65. doi:10.1056/NEJMoa1214609

13. Mayer SA, Brun NC, Begtrup K, Broderick J, Davis S, Diringer MN, et al. Efficacy and safety of recombinant activated factor VII for acute intracerebral hemorrhage. N Engl J Med (2008) 358:2127-37. doi:10.1056/ NEJMoa0707534

14. Mayer SA, Davis SM, Skolnick BE, Brun NC, Begtrup K, Broderick JP, et al. Can a subset of intracerebral hemorrhage patients benefit from hemostatic data collection, data analysis, data interpretation, or writing of the report. TP received honoraria as speakers for Genzyme, Boehringer Ingelheim, and Bayer. He is on the advisory board for Genzyme on Fabry Disease.

\section{VISTA-ICH STEERING COMMITTEE}

D. F. Hanley (Chair), K. Butcher, B. Gregson, S. Davis, K. R. Lees, P. Lyden, S. Mayer, K. Muir, and T. Steiner.

therapy with recombinant activated factor VII? Stroke (2009) 40:833-40. doi:10.1161/STROKEAHA.108.524470

15. Flemming KD, Wijdicks EF, Li H. Can we predict poor outcome at presentation in patients with lobar hemorrhage? Cerebrovasc Dis (2001) 11:183-9. doi:10.1159/000047636

16. Takahashi O, Cook EF, Nakamura T, Saito J, Ikawa F, Fukui T. Risk stratification for in-hospital mortality in spontaneous intracerebral haemorrhage: a classification and regression tree analysis. QJM (2006) 99:743-50. doi:10.1093/ qjmed/hcl107

17. Breiman L, Friedman JH, Olshen RA, Stone CJ. Classification and Regression Trees. Belmont, CA: Wadsworth (1983).

18. Ali M, Bath P, Brady M, Davis S, Diener HC, Donnan G, et al. Development, expansion, and use of a stroke clinical trials resource for novel exploratory analyses. Int J Stroke (2012) 7:133-8. doi:10.1111/j.1747-4949. 2011.00735.x

19. Han J, Kamber M. Data Mining. Concepts and Techniques. San Francisco: Morgan Kaufmann Publishers (2006).

20. Galimberti G, Soritti G, Di Maso M. Classification trees for ordinal responses in R: the rpartScore package. J Stat Softw (2012) 47:1-25. doi:10.18637/jss v047.i10

21. Specogna AV, Patten SB, Turin TC, Hill MD. The reliability and sensitivity of the National Institutes of Health Stroke Scale for spontaneous intracerebral hemorrhage in an uncontrolled setting. PLoS One (2013) 8:e84702. doi:10.1371/journal.pone.0084702

22. Saver JL. Novel end point analytic techniques and interpreting shifts across the entire range of outcome scales in acute stroke trials. Stroke (2007) 38:3055-62. doi:10.1161/STROKEAHA.107.488536

23. Hosmer DW, Lemeshow S. Applied Logistic Regression. New York: John Wiley \& Sons (2000).

24. Demchuk AM, Dowlatshahi D, Rodriguez-Luna D, Molina CA, Blas YS, Dzialowski I, et al. Prediction of haematoma growth and outcome in patients with intracerebral haemorrhage using the CT-angiography spot sign (PREDICT): a prospective observational study. Lancet Neurol (2012) 11:307-14. doi:10.1016/S1474-4422(12)70038-8

25. Lyden PD, Shuaib A, Lees KR, Davalos A, Davis SM, Diener HC, et al. Safety and tolerability of NXY-059 for acute intracerebral hemorrhage: the CHANT Trial. Stroke (2007) 38:2262-9. doi:10.1161/STROKEAHA.106.472746

26. Coste J, Walter E, Wasserman D, Venot A. Optimal discriminant analysis for ordinal responses. Stat Med (1997) 16:561-9. doi:10.1002/ (SICI)1097-0258(19970315)16:5<561::AID-SIM431>3.3.CO;2-3

27. Harrell FE Jr, Margolis PA, Gove S, Mason KE, Mulholland EK, Lehmann D, et al. Development of a clinical prediction model for an ordinal outcome: the World Health Organization multicentre study of clinical signs and etiological agents of pneumonia, sepsis and meningitis in young infants. WHO/ARI Young Infant Multicentre Study Group. Stat Med (1998) 17:909-44. doi:10.1002/ (SICI)1097-0258(19980430)17:8<909::AID-SIM753>3.0.CO;2-O

28. Hemphill JC III, Newman J, Zhao S, Johnston SC. Hospital usage of early do-not-resuscitate orders and outcome after intracerebral hemorrhage. Stroke (2004) 35:1130-4. doi:10.1161/01.STR.0000125858.71051.ca

29. Churilov L, Arnup S, Johns H, Leung T, Roberts S, Campbell BC, et al. An improved method for simple, assumption-free ordinal analysis of the modified Rankin Scale using generalized odds ratios. Int J Stroke (2014) 9:999-1005. doi:10.1111/ijs.12364 
30. Schellinger PD, Shuaib A, Kohrmann M, Liebeskind DS, Jovin T, Hammer MD, et al. Reduced mortality and severe disability rates in the SENTIS trial. AJNR Am J Neuroradiol (2013) 34:2312-6. doi:10.3174/ ajnr.A3613

31. MacIsaac RL, Khatri P, Bendszus M, Bracard S, Broderick J, Campbell B, et al. A collaborative sequential meta-analysis of individual patient data from randomized trials of endovascular therapy and tPA vs. tPA alone for acute ischemic stroke: thrombectomy and tPA (TREAT) analysis: statistical analysis plan for a sequential meta-analysis performed within the VISTAEndovascular collaboration. Int J Stroke (2015) 10(Suppl A100):136-44. doi:10.1111/ijs.12622

32. O'Donnell MJ, Fang J, D'Uva C, Saposnik G, Gould L, McGrath E, et al. The PLAN score: a bedside prediction rule for death and severe disability following acute ischemic stroke. Arch Intern Med (2012) 172:1548-56. doi:10.1001/2013. jamainternmed.30
33. Lees KR, Khatri P; STAIR IX Collaborators. Stroke treatment academic industry roundtable recommendations for individual data pooling analyses in stroke. Stroke (2016) 47:2154-9. doi:10.1161/STROKEAHA.116.012966

Conflict of Interest Statement: The authors declare that the research was conducted in the absence of any commercial or financial relationships that could be construed as a potential conflict of interest.

Copyright (๑) 2017 Phan, Chen, Beare, Ma, Clissold, Van Ly, Srikanth and the VISTA-ICH Collaboration. This is an open-access article distributed under the terms of the Creative Commons Attribution License (CC BY). The use, distribution or reproduction in other forums is permitted, provided the original author(s) or licensor are credited and that the original publication in this journal is cited, in accordance with accepted academic practice. No use, distribution or reproduction is permitted which does not comply with these terms. 